\title{
Identificación molecular de aislados de Trichoderma spp. y su actividad promotora en Solanum lycopersicum L.
}

\author{
Molecular identification of isolates of Trichoderma spp. and promoting \\ activity in Solanum lycopersicum L.
}

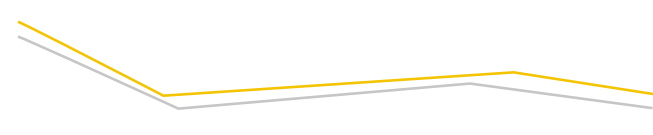

Felicia Amalia Moo-Koh*, Jairo Cristóbal-Alejo*®, Arturo ReyesRamírez*, José María Tun Suárez*, Marcela Gamboa-Angulo**

Moo-Koh, F. A., Cristóbal-Alejo, J., Reyes-Ramírez, A., Tun Suárez, J. M., \& GamboaAngulo, M. (2017). Identificación molecular de aislados de Trichoderma spp. y su actividad promotora en Solanum lycopersicum L. Investigación y Ciencia de la Universidad Autónoma de Aguascalientes, 25(71), 5-11.

RESUMEN

El presente estudio se dirigió al aislamiento e identificación de especies de Trichoderma y su estimación de promoción de crecimiento vegetal en plántulas de Solanum lycopersicum L. cv. Río Grande. Se aislaron cuatro cepas; dos se identificaron como T. ghanense (Th26-52 y Th02-04), una como $T$. citrinoviride (Th33-58) y otra como $T$. harzianum (Th33-59). Los resultados de promoción en plántulas de $S$. lycopersicum en concentraciones de $10^{6}$ conidios $\cdot \mathrm{ml}^{-1}$ más fertilización química a $50 \%$, mostraron diferencias estadísticas significativas entre tratamientos $(p \leq .01)$. Los tratamientos con Trichoderma spp. incrementaron de 24.28 a $38.53 \%$ la altura de plántula, de 2.29 a $14.09 \%$ el diámetro de tallo, de 8.48 a $16.54 \%$ la longitud de raíz, de

Palabras clave: Trichoderma ghanense; Trichoderma citrinoviride; Trichoderma harzianum; biofertilizantes; saprófitos; plántulas.

Keywords: Trichoderma ghanense; Trichoderma citrinoviride; Trichoderma harzianum; biofertilizer; saprophytic; seedlings.

Recibido: 5 de octubre de 2016, aceptado: 2 de febrero de 2017

* División de Estudios de Posgrado e Investigación, Instituto Tecnológico de Conkal. Avenida Tecnológico s/n, C. P. 97345, Conkal, Yucatán, México. Correo electrónico: famk22@hotmail.com; jairoca54@hotmail.com; arte_rey@hotmail.com; tun@colpos.mx

* Unidad de Biotecnología, Centro de Investigación Científica de Yucatán, A. C. Calle 43 No. 130, Colonia Chuburná de Hidalgo, C. P. 97205, Mérida, Yucatán, México. Correo electrónico: mmarcela@cicy.mx

$\otimes \quad$ Autor para correspondencia
0.37 a $2.68 \%$ el volumen radical y de 0 a $23.07 \%$ la biomasa seca total, con respecto al testigo que consistió únicamente de fertilización química a $50 \%$. T. ghanense (Th26-52) mostró potencial para mejorar el crecimiento de plántulas en S. lycopersicum.

\section{ABSTRAC}

The objective of this study was to isolate, identify and estimate the promoting plant growth of species of Trichoderma. Four strains were isolated, two were identified as T. ghanense (Th26-52 and Th02-04) and as T. citrinoviride (Th33-58) and once as T. harzianum (Th33-59). Promotion results in seedlings of Solanum lycopersicum L. CV. Rio Grande at concentrations of $10^{6}$ conidios $\mathrm{ml}^{-1}$ and $50 \%$ chemical fertilizer showed significant differences between treatments $(p \leq .01)$. Treatment with Trichoderma spp. increased from 24.28 to $38.53 \%$ the seedling height, 2.29 to $14.09 \%$ the stem diameter, 8.48 to $16.54 \%$ the root length, 0.37 to $2.68 \%$ the root volume and 0 to $23.07 \%$ the total dry biomass compared to the control with chemical fertilization 50\%. T. ghanense (Th26-52) showed potential to improve seedling growth in S. lycopersicum.

\section{INTRODUCCIÓN}

En plantas, el efecto benéfico de la inoculación de especies de Trichoderma se consigue cuando mantienen una estable colonización producto de una interacción química que se refleja en la germinación de semillas, donde la producción de enzimas líticas de Trichoderma actúa en el epispermo 
IIVESTIGACIÓn Y CIERCIA DE LA UNIVERSIDAD AUTÓNOMA DE RGUASCALIERTES de éstas (López-Bucio, Pelagio-Flores, \& RiveraEstrella, 2015). En plántulas, Trichoderma promueve el desarrollo de tejidos meristemáticos primarios, los cuales aumentan la altura de plántula, el peso y el desarrollo de las raíces laterales que mejoran la asimilación de nutrientes y con ello la acumulación de antocianinas en hojas (Contreras-Cornejo, Macías-Rodríguez, Beltrán-Peña, Herrera-Estrella, \& López-Bucio, 2011; Marín-Guirao, Rodríguez-Romera, Lupión-Rodríguez, Camacho-Ferre, \& Tello-Marquina, 2016).

La inoculación de Trichoderma tiene efecto en el crecimiento y desarrollo de Arabidopsis thaliana L. y en cultivos de importancia agrícola como Passiflora edulis var. flavicarpa Degener (Cubillos-Hinojosa, Valero, \& Mejía, 2009), Solanum lycopersicum L., Theobroma cacao L., Salvia hispanica L., Zea mays L., Nicotina tabacum L., Latuca sativa L. y Raphanus sativus L., Capsicum annuum L. var. longum y Capsicum chinense Jacq. (Candelero et al., 2015).

La plasticidad biológica de Trichoderma le confiere un potencial como biofertilizante (Leelavathi, Vani, \& Reena, 2014) y antagonista contra fitopatógenos, son los hongos más estudiados de la rizósfera y comercialmente disponibles para su aplicación como enmiendas del suelo (Kumar Solanki e† al., 2011). Sin embargo, se ha visto que la utilización de aislados adaptados a las condiciones ambientales de donde se aíslan tiene más éxito durante las evaluaciones para estos propósitos (Candelero et al., 2015; Marín-Guirao, Rodríguez-Romera, LupiónRodríguez, Camacho-Ferre, \& Tello-Marquina, 2016).

Los objetivos de esta investigación fueron aislar, identificar y estimar la capacidad promotora de crecimiento vegetal de aislados de Trichoderma en plántulas de $S$. Iycopersicum y relacionar de manera filogenética las especies identificadas molecularmente.

\section{MATERIALES Y MÉTODOS}

Para obtener los aislados de Trichoderma se realizó un muestreo directo de suelo, con y sin actividad agrícola, en el estado de Yucatán. Se utilizó la técnica de partículas de suelo sin lavar (Bills, Christensen, Powell, \& Thorn, 2004). La identificación preliminar se basó según fiálides, conidios, color y forma de crecimiento micelial.
La identificación molecular de Trichoderma spp. se realizó a partir de cultivos monospóricos (Zhenyu, Man, Xinzheng, Libo, \& Li, 2014) se extrajo el ADN con el kit ZR Fungal/Bacterial DNA MiniPrep ${ }^{T M}$. Se amplificó la región ITS 1-5.8s-ITS2 del ADN ribosomal mediante la reacción en cadena de polimerasa (PCR) usando los iniciadores ITS1 e ITS4 (White, Bruns, Lee, \& Taylor, 1990) bajo las siguiente condiciones: desnaturalización inicial de $5 \mathrm{~min}$ a $94^{\circ} \mathrm{C}$, seguido de 30 ciclos (desnaturalización a $95^{\circ} \mathrm{C}$ por $1 \mathrm{~min}$, alineación a $54^{\circ} \mathrm{C}$ por 30 s y una extensión de 1 min a $72^{\circ} \mathrm{C}$ ) con una extensión final de 5 min a $72^{\circ} \mathrm{C}$ (MooKoh et al., 2014; White et al., 1990). Los productos de PCR se enviaron a secuenciar a la empresa Macrogen USA. Las secuencias se compararon con la base de datos del Banco de Genes del National Center for Biotechnology Information (s. f.) en el programa Basic Local Alignment Search Tool (BLAST) y se elaboró un árbol filogenético con el software MEGA versión 6 con el método Neighbor-Joining (Tamura, Stecher, Peterson, Filipski, \& Kumar, 2013).

Para evaluar la promoción de crecimiento en S. Iycopersicum, se prepararon semilleros de tomate del tipo Saladette cv. Río Grande como modelo de estudio, preparados con sustrato comercial Cosmopeat ${ }^{\circledR}$ mezclado previamente con agrolita (50:50 p/p), con una inoculación inicial de $10^{6}$ conidios $\mathrm{ml}^{-1}$ de cada uno de los hongos aislados (Th02-04, Th26-52, Th33-58 y Th33-59); posteriormente se realizaron dos inoculaciones fúngicas, a los 8 y 15 días después de la germinación. A partir de los 8 días después de la misma, las plántulas se mantuvieron con riego diario y fertilización química a $50 \%$ (20N-20P20K, $1 \mathrm{~g} \mathrm{t}^{-1}$ ). El testigo sin inoculación de Trichoderma consistió solo en fertilización química (FQ) a $50 \%$ (Candelero et al., 2015). El efecto promotor de los aislados fúngicos se realizó a los 25 días después de la germinación; cuando las plántulas tuvieron las características agronómicas para un trasplante definitivo, de por lo menos $15 \mathrm{~cm}$ de altura y $3 \mathrm{~mm}$ de diámetro de tallo (Cai et al., 2015). Se consideraron 21 plantas como unidad experimental por tratamiento, con cuatro repeticiones.

Los tratamientos se distribuyeron en un diseño experimental completamente al azar, en condiciones de invernadero. Las variables respuesta fueron: altura de plántula $(\mathrm{cm})$, diámetro de tallo $(\mathrm{mm})$, longitud de raíz $(\mathrm{cm})$, volumen radical $(\mathrm{ml})$ y biomasa seca total (g). Con los datos obtenidos se realizaron análisis de varianza y para la comparación 


\section{InVESTIGACIÓn Y CIERCIA DE LA UחIVERSIDAD AUTÓnOMA DE RGUASCALIETTES}

de medias se consideró el método de Tukey ( $p \leq 0.05)$. Con el propósito de obtener el mejor aislado fúngico en la promoción de crecimiento, se realizó un análisis de conglomerado donde se agruparon las variables estimadas con los promedios mayores, con los mismos se formó un testigo positivo, y los promedios más bajos constituyeron el testigo negativo. El análisis estadístico se realizó con el paquete Statistical Analysis System (SAS) ver. 9.4.

Tabla 1

Aislados de Trichoderma obtenidos de localidades del estado de Yucatán

\begin{tabular}{|c|c|c|c|c|c|}
\hline $\begin{array}{l}\text { Clave de } \\
\text { aislado }\end{array}$ & Origen & Tipo de suelo & Latitud & Longitud & $\begin{array}{c}\text { Altitud } \\
\text { (m.s.n.m.) }\end{array}$ \\
\hline Th02-04 & Tizimín & SCAA* & $21^{\circ} 08^{\prime} 33^{\prime \prime} \mathrm{N}$ & $88^{\circ} 09^{\prime} 53^{\prime \prime} \mathrm{O}$ & 20 \\
\hline Th26-52 & Tahdziú & SCAA & $20^{\circ} 12^{\prime} 08^{\prime \prime} \mathrm{N}$ & $88^{\circ} 56^{\prime} 35^{\prime \prime} \mathrm{O}$ & 32 \\
\hline Th33-58 & Ticul & SSAA** & $20^{\circ} 23^{\prime} 43^{\prime \prime} \mathrm{N}$ & $89^{\circ} 32^{\prime} 02^{\prime \prime} \mathrm{O}$ & 25 \\
\hline Th33-59 & Ticul & SSAA & $20^{\circ} 23^{\prime} 43^{\prime \prime} \mathrm{N}$ & $89^{\circ} 32^{\prime} 02^{\prime \prime} \mathrm{O}$ & 25 \\
\hline
\end{tabular}

El análisis de las secuencias genéticas permitió 99\% de homología para los aislados Th02-04 y Th26-52 con dos secuencias diferentes registradas en la base de datos del NCBI con claves de acceso JN564003 y LN84670; sin embargo, coincidieron con la misma especie: T. ghanense. El aislado Th33-58 coincidió en $99 \%$ con Hypocrea schweinitzii al alinearse con la secuencia FJ605263, mientras que el Th33-59 tuvo $100 \%$ de homología con $\mathrm{H}$. lixii en la secuencia FR872742. En todos los casos se obtuvieron resultados significativos con puntuaciones máximas de $>500$ y con valor de $\mathrm{E}<0.0$.

En la construcción del árbol filogenético se visualizó la relación genética inter e intraespecífica. Se observó en las especies de T. ghanense (Th0204 y Th26-52), aunque presentaron una similitud de $99 \%$, se agruparon de manera independiente con respecto a las secuencias del NCBI. H. schweinitzii (Th33-58) se agrupó con el clado de $H$. schweinitzii (T. citrinoviride) y para el aislado Th33-59, con el de $H$. lixii (T. harzianum) (figura 1).

\section{Efecto de Trichoderma spp. en el crecimiento de $S$. lycopersicum}

Los análisis de varianza mostraron diferencias estadísticamente significativas ( $p \leq 0.01$ ) entre tratamientos. En general, los tratamientos con las especies $T$. ghanense (Th26-52), T. citrinoviride (Th33-58) y T. harzianum (Th33-59) presentaron los mayores promedios

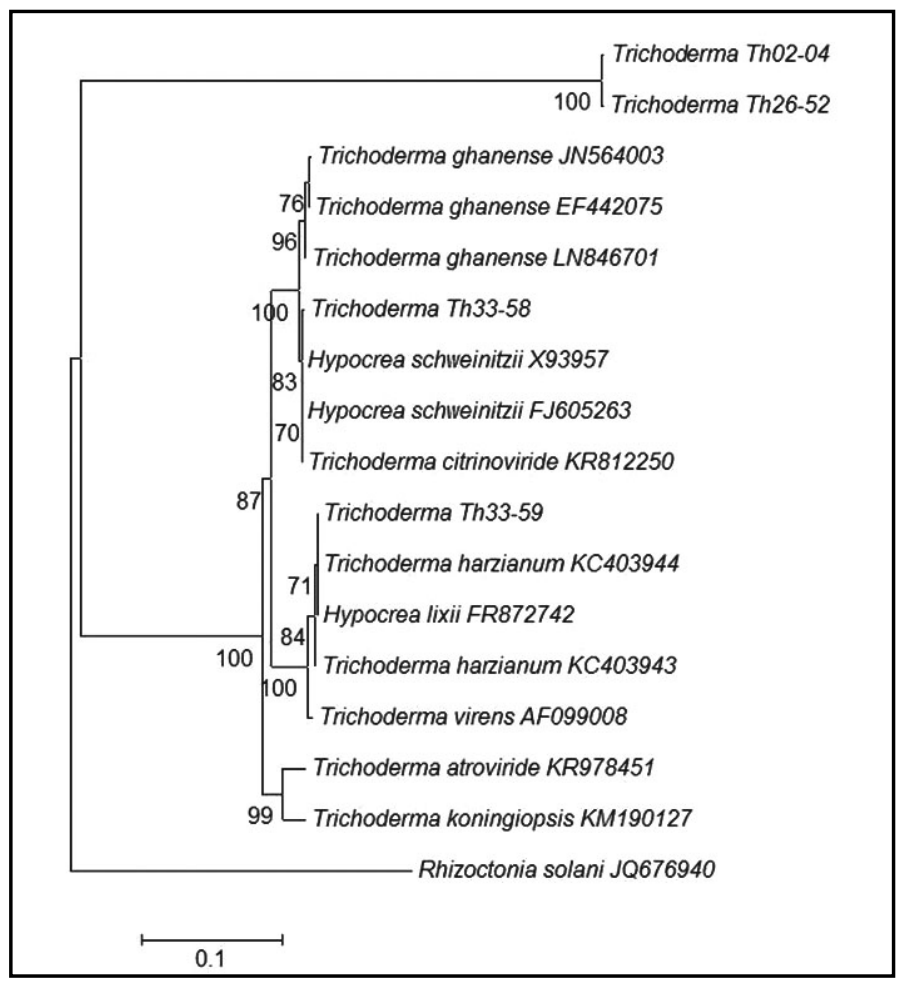

Figura 1. Relación filogenética de cuatro aislados de Trichoderma spp. El árbol se construyó a partir del análisis de secuencias ITS por el método Neighbor-Joining usando el modelo Tamura et al. (2013).

en las variables altura de plántula (figura 2), diámetro del tallo y biomasa seca total; T. ghanense (Th26-52) 


\section{INVESTIGAGIÓก Y CIECEIA DE LA UNIVERSIDAD AUTÓNOMA DE AGUASCALIERTES

presentó los mayores incrementos con ganancias de $38.53,14.09$ y $23.07 \%$ en relación con el tratamiento testigo FQ $50 \%$. En longitud de raíz $T$. citrinoviride (Th33-58) causó mayor efecto con ganancias de $16.54 \%$, mientras que en el volumen radical la aplicación de T. harzianum (Th33-59) lo incrementó con 2.68\%. La comparación de medias (Tukey, $p \leq 0.05$ ) en la altura de plántula detectó diferencias entre las especies fúngicas y el testigo $\mathrm{FQ} \mathrm{50 \% ;} \mathrm{en} \mathrm{el} \mathrm{diámetro}$ de tallo, solo T. ghanense (Th26-52) no superó al testigo FQ 50\%; mientras que en la longitud de raíz, T. harzianum (Th33-59) y T. citrinoviride (33-58) lo superaron; en el volumen radical y en biomasa seca total, solo $T$. harzianum (Th33-59) fue mayor (tabla 2).

Tabla 2

Efecto de la inoculación de Trichoderma spp. en la promoción de crecimiento vegetal para la producción de plántulas de S. lycopersicum cv. Río Grande

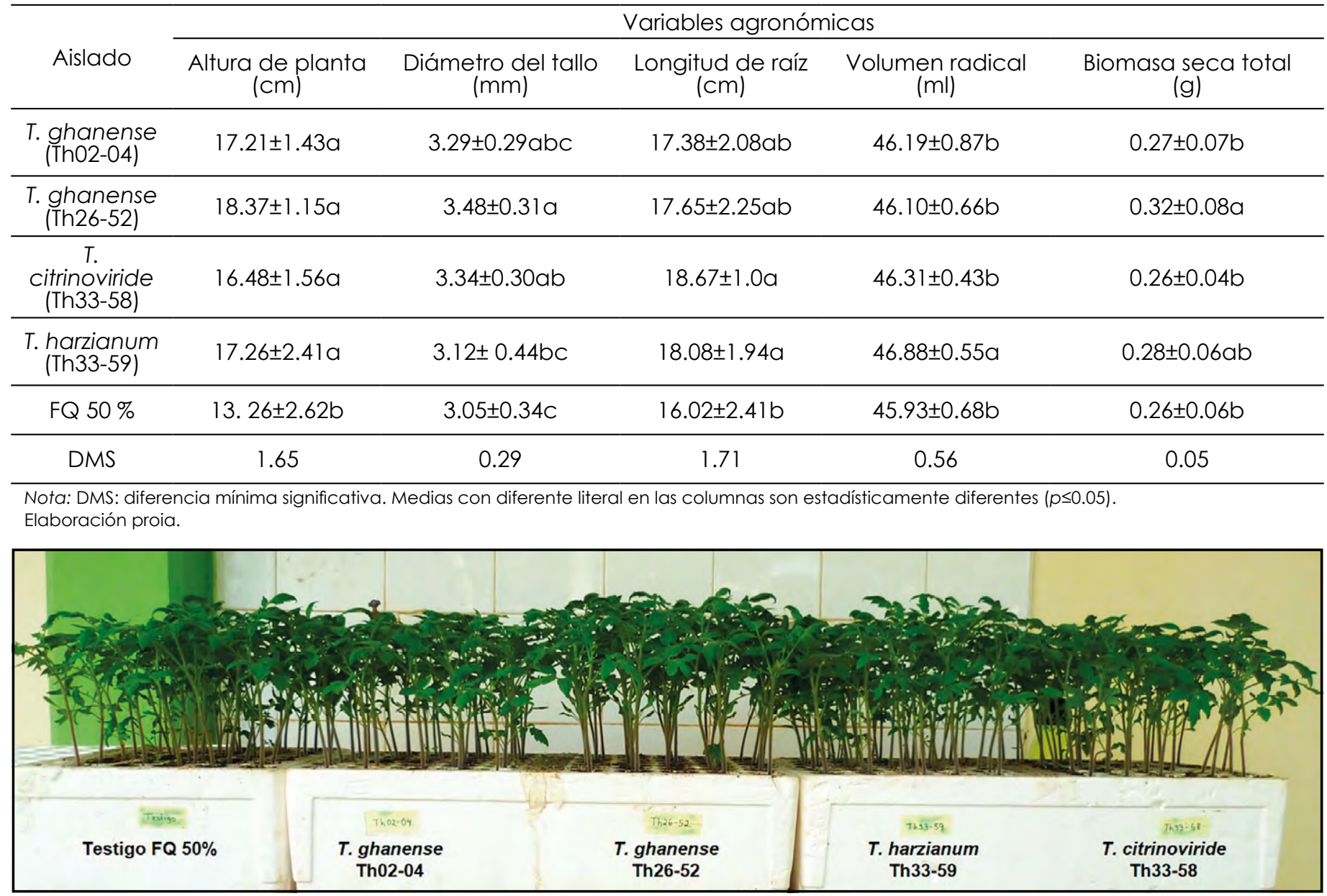

Figura 2. Efecto de la inoculación de Trichoderma spp. en la promoción de crecimiento de plántulas de S. Iycopersicum. Fotografía proporcionada por los autores.

Con base en el análisis de conglomerados, $T$. ghanense (Th26-52) fue el mejor tratamiento para promover el crecimiento vegetal, ya que su similitud fue la más cercana al testigo positivo (figura 3).

\section{DISCUSIÓN}

Estudios recientes sugieren que las relaciones entre Trichoderma (anamorfo) e Hypocrea (teleomorfo) son incorrectas; sin embargo, existen especies de Trichoderma cuyo teleomorfo se encuentra descrito en Hypocrea (Bissett, Gams, Jaklitsch, \& Samuels, 2015). Así, en aquellos hongos en los que se conoce la reproducción sexual y asexual se recomienda usar el género de uso común y más antiguo nombrado, cuando se encuentran representados por un aislado tipificado y cuando su secuencia de ADN se encuentra en el banco de genes. Esto 


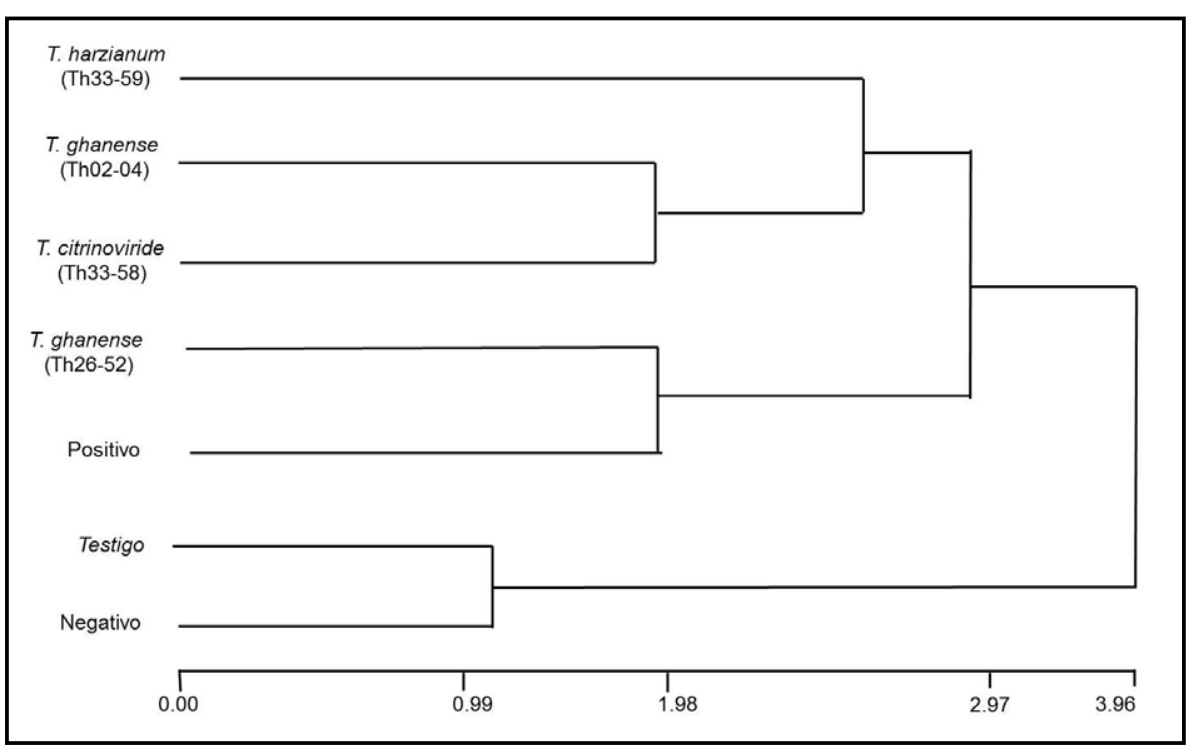

Figura 3. Dendrograma del análisis de conglomerados del efecto de la inoculación de Trichoderma spp. en la promoción de crecimiento vegetal en plántulas de $S$. lycopersicum cv. Río Grande.

Elaboración propia.

significa que el género Trichoderma es el adecuado para determinadas especies, cuyo teleomorfo se encuentra dentro del género Hypocrea; por tanto, las especies $H$. schweinitzii (Th33-58) e H. lixii (Th3359) pueden nombrarse como $T$. citrinoviride y $T$. harzianum, respectivamente. El hecho de que se haya identificado a Trichoderma molecularmente como Hyprocrea solo fue esencial para aclarar y confirmar la biología y plasticidad de este hongo (Jaklitsch, \& Voglmayr, 2014).

El desarrollo de técnicas moleculares para el análisis de secuencias filogenéticas ha contribuido a la comprensión de la evolución de Hypocrea/ Trichoderma y ha ayudado a mostrar el vínculo entre anamorfos particulares y teleomorfos. En aquellas especies estrechamente relacionadas de Trichoderma se ha utilizado el gen factor de elongación 1-alfa (EF-l $\alpha)$; sin embargo, estudios demuestran que el análisis de las secuencias ITS de los genes ribosomales han sido determinantes para distinguir un hongo de otro (Moo-Koh et al., 2014). Cuando se realiza el análisis y existe una identidad y una cobertura de $99 \%$ con las secuencias del banco de genes se considera a organismos de la misma especie (Pavone Maniscalco, \& Dorta, 2015), como ocurrió con T. ghanense (Th02-04 y Th26-52) y T. citrinoviride (Th33-58) (Jaklitsch, \& Voglmayr, 2014).
Los estudios sobre la biodiversidad de hongos microscópicos en las zonas tropicales son escasos. Con el presente estudio se contribuye al conocimiento de dos aislados de T. ghanense (Th02-04 y Th2652) procedentes de distintas zonas geográficas, lo que pone en evidencia el papel relativo de la actividad humana que favorece el establecimiento de especies de Trichoderma e interactúa con la comunidad endémica y el ambiente. La exposición de especies de Trichoderma en diversas zonas y las condiciones donde se desarrollan establecen una diversidad intraespecífica (Hermosa et al., 2013).

Las investigaciones de selección ayudan a determinar el potencial de aislados de Trichoderma, esto se demostró con los aislados de T. ghanense (Th02-04 y Th26-52), los cuales provienen de diferentes localidades y de suelos con actividad agrícola; sin embargo, presentaron diferente actividad promotora de crecimiento.

El potencial de Trichoderma como promotor de crecimiento vegetal se ha reportado en estudios como el de Donoso, Lobos y Rojas (2008), que en Pinus radiata D. Don con aplicaciones de T. harzianum, en combinación con composta, incrementaron la altura de la planta de 24.4 a $29.3 \mathrm{~cm}$; es decir, $20.1 \%$, de 5.9 a $7.7 \mathrm{~g}$, equivalente a $30.5 \%$ en la biomasa 
IIVESTIGACIÓn Y CIERCIA DE LA UNIVERSIDAD AUTÓNOMA DE RGUASCALIERTES seca total, y de 4.9 a $6.6 \mathrm{~g}, 34.7 \%$ en la aérea en comparación con los tratamientos que no incluyeron composta. La adición de composta es la opción para el mantenimiento de poblaciones fúngicas, mejora las condiciones del suelo y estabiliza el rendimiento de los cultivos (Cai et al., 2015).

En plántulas de $P$. edulis var. Flavicarpa, Cubillos-Hinojosa et al. (2009) obtuvieron incrementos significativos al inocular un aislado de T. harzianum, el mismo tuvo efectos sobre el diámetro del tallo, longitud de raíz y peso seco total con incrementos de $57.1 \%$, $63 \%$ y $125 \%$, en su orden, en relación con una especie comercial, lo que confirmó la ventaja de aplicar aislados locales. En tomate, Zhenyu et al. (2014) con T. harzianum TL-1, incrementaron el área foliar (97.34\%), la altura de planta (61.22\%), el peso fresco (167.98\%) y el peso seco (131.82\%). En Capsicum chinense Jacq., Candelero et al. (2015) con aislados de $T$. virens, mostraron efectos significativos en altura de planta con incrementos de 55.57 y 47.62\%; sin embargo, un aislado de T. harzianum lo consideraron como el mejor al causar mayor volumen radical con ganancias de $84.61 \%$ en relación con el tratamiento sin aislado fúngico. La explica- ción de la actividad promotora de crecimiento de Trichoderma es su capacidad para producir ácido indolácetico. El mismo actúa como catalizador de tejidos meristemáticos primarios y favorece la división celular o suprime concentraciones de ácido abscísico (Martínez-Medina, Alguacil, Pascual, \& Van Wees, 2014; Vinale et al., 2013).

CONCLUSIONES

Se identificaron tres especies: T. ghanense (Th26-52 y Th02-04), T. citrinoviride (Th33-58) y T. harzianum (Th33-59), con actividad promotora de crecimiento vegetal.

La especie con mejor efecto promotor de crecimiento en plántulas de $S$. lycopersicum fue $T$. ghanense (Th26-52).

\section{Agradecimientos}

Los autores desean agradecer al proyecto "Contribución al estudio de aislados de Trichoderma spp. con aplicación agrícola", apoyado por el TECNM, con clave 5726.16 P.

REFERENCIAS

- Bills, G. F., Christensen, M., Powell, M., \& Thorn, G. (2004). Saprobic soil fungi. En G. Mueller, F. Bills, \& M. Foster (Eds.), Biodiversity of fungi: Inventory and monitoring methods (pp. 271-302). Amsterdam, Netherlands: Academic Press.

- Bissett, J., Gams, W., Jaklitsch, W., \& Samuels, G. J. (2015). Accepted Trichoderma names in the year 2015. International Mycological Association, 6(2), 263-295. doi:10.5598/ imafungus.2015.06.02.02

- Cai, F., Chen, W., Wei, Z., Pang, G., Li, R., Ran, W., \& Shen, Q. (2015). Colonization of Trichoderma harzianum strain SQR-T037 on tomato roots and its relationship to plant growth, nutrient availability and soil microflora. Plant and Soil, 388(1), 337-350. doi:10.1007/s $11104-014-2326-z$

- Candelero, D. J., Cristóbal, A. J., Reyes, R. A., Tun, S. J. M., Gamboa, A. M. M., \& Ruíz, S. E. (2015). Trichoderma spp. promotoras del crecimiento en plántulas de Capsicum chinense Jacq. y antagónicas contra Meloidogyne incognita. Revista Internacional de Botánica Experimental фYTON, 84, 113-119. Recuperado de http://www.revistaphyton.fundromuloraggio.org.ar/vol84-1/Candelero.pdf

- Contreras-Cornejo, H. A., Macías-Rodríguez, L., Beltrán-Peña, E., Herrera-Estrella, A., \& López-Bucio, J. (2011). Trichoderma- induced plant immunity likely involves both hormonal- and camalexin-dependent mechanisms in Arabidopsis thaliana and confers resistance against necrotrophic fungi Botrytis cinerea. Plant Signaling \& Behavior, 6(10), 1554-1563. doi:10.4161/psb.6.10.17443

- Cubillos-Hinojosa, J., Valero, N., \& Mejía, L. (2009). Trichoderma harzianum como promotor del crecimiento vegetal del maracuyá (Passiflora edulis var. flavicarpa Degener). Agronomía Colombiana, 27(1), 81-86. Recuperado de http:// www.redalyc.org/articulo.oa?id $=180314730011$

- Donoso, E., Lobos, G. A., \& Rojas, N. (2008). Efecto de Trichoderma harzianum y compost sobre el crecimiento de plántulas de Pinus radiata en vivero. Bosque, 29(1), 52-57. Recuperado de http://www. scielo.cl/pdf/bosque/v29n1/art06.pdf

- Hermosa, R., Rubio, M. B., Cardoza, R. E., Nicolás, C., Monte, E., \& Gutiérrez, S. (2013). The contribution of Trichoderma to balancing the costs of plant growth and defense. International Microbiology, 16, 69-80. doi:10.2436/20.1501.01.181

- Jaklitsch, W. M., \& Voglmayr, H. (2014). New combinations in Trichoderma (Hypocreaceae, Hypocreales). Mycotaxon, 126, 143-156. doi:10.5248/126.143 


\section{IIVESTIGAGIÓn Y CUERGIA DE LA UחIVERSIDAD AUTÓNOMA DE RGUASCALIERTES}

- Kumar Solanki, M., Singh, N., Kumar Singh, R., Singh, P., Srivastava, A. K., Kumar, S., ... Arora, D. K. (2011). Plant defense activation and management of tomato root rot by a chitinfortified Trichoderma/Hypocrea formulation. Phytoparasitica, 39, 471-481. doi:10.1007/s12600-011-0188-y

- Leelavathi, M. S, Vani, L., \& Reena, P. (2014). Antimicrobial activity of Trichoderma harzianum against bacteria and fungi. International Journal of Current Microbiology and Applied Sciences, 3(1), 96-103. Recuperado de http://www.ijcmas. com/vol-3-1/M.S.Leelavathi,\%20et\%20al.pdf

- López-Bucio, J., Pelagio-Flores, R., \& Herrera-Estrella, A. (2015). Trichoderma as biostimulant: exploiting the multilevel properties of a plant beneficial fungus. Scientia Horticulturae, 196, 109-123. Recuperado de http://dx.doi.org/10.1016/j. scienta.2015.08.043

- Marín-Guirao, J. I., Rodríguez-Romera, P., Lupión-Rodríguez, B., Camacho-Ferre, F., \& Tello-Marquina, J. C. (2016). Effect of Trichoderma on horticultural seedlings' growth promotion depending on inoculum and substrate type. Journal of Applied Microbiology, 121 (4), 1095-1 102. doi:10.1111/jam.13245

- Martínez-Medina A., Alguacil, M. M., Pascual, J. A., \& Van Wees, S. C. (2014). Phytohormone profiles induced by Trichoderma isolates correspond with their biocontrol and plant growthpromoting activity on melon plants. Journal of Chemical Ecology, 40(7), 804-815. doi:10.1007/s10886-014-0478-1

- Moo-Koh, F. A., Alejo, J. C., Reyes-Ramírez, A., Tun-Suárez, J. M., Sandoval-Luna, R., \& Ramírez-Pool, J. A. (2014). Actividad in vitro del extracto acuoso de Bonellia flammea contra hongos fitopatógenos. Agrociencia, 48(8), 833-845. Recuperado de http://www.scielo.org.mx/pdf/agro/v48n8/v48n8a6.pdf
- National Center for Biotechnology Information. Basic Local Alignment Search Too (BLAST). Recuperado de https://www. ncbi.nlm.nih.gov/

- Pavone Maniscalco, D., \& Dorta, B. (2015). Diversidad del hongo Trichoderma spp. en plantaciones de maíz de Venezuela. Asociación Interciencia, 40(1), 23-31. Recuperado de http://www.redalyc.org/articulo.oa?id=33933115004

- Tamura, K., Stecher, G., Peterson, D., Filipski, A., \& Kumar, S. (2013). MEGA6: Molecular Evolutionary Genetics Analysis version 6.0. Molecular Biology and Evolution, 30(12), 2725-2729. doi:10.1093/molbev/mst197

- Vinale, F., Nigro, M., Sivasithamparam, K., Flematti, G., Ghisalberti, E. L., Ruocco, M., ... Lorito, M. (2013). Harzianic acid: a novel siderophore from Trichoderma harzianum. FEMS Microbiol Lett, 347(2), 123-129. doi:10.1111/1574-6968.12231

- White, T. J., Bruns, T., Lee, S., \& Taylor, J. (1990). Amplification and direct sequencing of fungal ribosomal RNA genes for phylogenetics. En M. A. Inns, D. H. Gelfand, J. J. Sninsky, \& T. J. White (Eds.), PCR Protocols: A guide to methods and applications (pp. 315-322). San Diego, CA: Academic Press.

- Zhenyu, S., Man, X., Xinzheng, G., Libo, T., \& Li, L. (2014). Primary study on biological control potential of Trichoderma harzianum TL-1. Plant Diseases and Pests, 5(2), 25-28. Recuperado de http://search.proquest.com/openview/d997794458cd80f3865 bd $15 \mathrm{fb} 5335546 / 1$ ? pq-origsite=gscholar 